\title{
On the heritability of inspection time and its covariance with IQ: a twin study
}

\author{
Michelle Luciano*, Glen A. Smith, Margaret J. Wright, Gina M. Geffen, \\ Laurie B. Geffen, Nicholas G. Martin
}

Queensland Institute of Medical Research, Brisbane, Queensland, Australia

University of Queensland, Brisbane, Queensland, Australia

Received 2 March 2001; accepted 2 March 2001

\begin{abstract}
Using the classical twin design, this study investigates the influence of genetic factors on the large phenotypic variance in inspection time (IT), and whether the well established IT-IQ association can be explained by a common genetic factor. Three hundred ninety pairs of twins (184 monozygotic, MZ; 206 dizygotic, DZ) with a mean age of 16 years participated, and 49 pairs returned approximately 3 months later for retesting. As in many IT studies, the pi figure stimulus was used and IT was estimated from the cumulative normal ogive. IT ranged from 39.4 to $774.1 \mathrm{~ms}$ $(159 \pm 110.1 \mathrm{~ms})$ with faster ITs (by an average of $26.9 \mathrm{~ms})$ found in the retest session from which a reliability of .69 was estimated. Full-scale IQ (FIQ) was assessed by the Multidimensional Aptitude Battery (MAB) and ranged from 79 to $145(111 \pm 13)$. The phenotypic association between IT and FIQ was confirmed $(-.35)$ and bivariate results showed that a common genetic factor accounted for $36 \%$ of the variance in IT and $32 \%$ of the variance in FIQ. The maximum likelihood estimate of the genetic correlation was -.63 . When performance and verbal IQ (PIQ \& VIQ) were analysed with IT, a stronger phenotypic and genetic relationship was found between PIQ and IT than with VIQ. A large part of the IT variance (64\%) was accounted for by a unique environmental factor. Further genetic factors were needed to explain the remaining variance in IQ with a small component of unique environmental variance present. The separability of a shared genetic factor influencing IT and IQ from the total genetic variance in IQ suggests that IT affects a specific
\end{abstract}

* Corresponding author. Queensland Institute of Medical Research, PO Royal Brisbane Hospital, Brisbane, Queensland 4029, Australia. Tel.: +61-7-3362-0218; fax: +61-7-3362-0101.

E-mail address: michellu@qimr.edu.au (M. Luciano). 
subcomponent of intelligence rather than a generalised efficiency. (C) 2001 Elsevier Science Inc. All rights reserved.

Keywords: IT; IQ; Heritability; Twins; Quantitative genetics

\section{Introduction}

In an ongoing study of the genetics of cognition, in adolescent Australian twins, inspection time (IT) was collected as part of a psychometric battery to probe processing speed. The principal aim is to identify genetic variants that influence individual differences in processing speed and working memory, both of which play a significant role in higher level functioning. A major focus is to investigate whether there is a genetic influence on the covariation among measures of these two basic abilities that accounts for a significant part of the genetic variance in intelligence. We will then use the correlated biological markers in future linkage and association analyses to search for quantitative trait loci (QTLs) influencing cognition.

In this paper, we estimate the heritability of the IT measure using the twin design and model fitting procedures that decompose the large phenotypic variance in IT into genetic and environmental components (Neale \& Cardon, 1992; Plomin, 1986). The classical twin design compares monozygotic (MZ) twin pairs who share $100 \%$ of their genes with dizygotic (DZ) twins who share roughly $50 \%$ of their segregating genes. If the causes of familial similarity are the additive genes (transmissible from parent to child), the correlation in performance of $\mathrm{MZ}$ co-twins is expected to be twice that of DZ co-twins. An underlying assumption is that environmental influences on cognition are equivalent in $\mathrm{MZ}$ and $\mathrm{DZ}$ twins (e.g., Kendler, Neale, Kessler, Heath, \& Eaves, 1993). The potential for genetic influence on individual differences in IT has not previously been investigated, and while several measures of information processing speed have been shown to be heritable (.20 to .76), the extent of the genetic influence is shown to be dependent on the specific processing measure used (Boomsma \& Somsen, 1991; McGue \& Bouchard, 1989; Vernon, 1989).

It has been argued by some authors that IT is a better measure of basic information processing than other elementary cognitive tasks due to the simplicity of the task and the elimination of the response time confound and speed-accuracy trade-off effects (Deary \& Stough, 1996). It has been vigorously pursued, with adaptations of the visual IT task to the auditory modality as a test of its validity (Deary, Caryl, Egan, \& Wight, 1989; Deary, Head, \& Egan, 1989). From a reductionist approach, it is claimed that IT causes IQ and not the reverse; empirical efforts to substantiate this theory have focused on exemplifying IT as a biological mechanism. One demonstration of this was the association between IT and event-related potentials: faster ITs were characterised by steeper P200 slopes (the positive peak $200 \mathrm{~ms}$ after stimulus onset) and higher intelligence test scores (Morris \& Alcorn, 1995).

Other studies have implicated a biological basis to the relationship between information processing speed and intelligence by demonstrating a genetic contribution to the association (Baker, Vernon, \& Ho, 1991; Ho, Baker, \& Decker, 1988; Petrill, Luo, Thompson, \& 
Detterman, 1996; Rijsdijk, Vernon, \& Boomsma, 1998). Although IT has not directly been investigated, various elementary cognitive tasks measuring reaction time or accuracy on rapid automatic naming and perceptual speed tests have been used. For instance, Baker et al. (1991) reported a heritability of .45 for a general reaction time factor (derived from 11 tests) and genetic correlations of 1 and .92, with verbal (VIQ) and performance IQs (PIQ), respectively. However, in an earlier study, it was found that, in addition to a common genetic factor, a common nonshared environment factor influenced an association between IQ and a rapid automatic naming factor, but not a symbol processing factor (Ho et al., 1988). The intercorrelation among elementary cognitive tasks is well known (Jensen, 1998), and therefore (like other basic component processes) IT is expected to demonstrate a genetic relationship with IQ, although the possibility of a common environmental relationship cannot be dismissed.

The current study, therefore, uses the twin method to partition the variance in IT among individuals into genetic and environmental components and examines the extent to which IT and psychometric intelligence are influenced by the same underlying factors. Following findings (Deary, 1993; Kranzler \& Jensen, 1989) of a stronger relationship between IT and PIQ than with VIQ, a contrast of these IQ scales with IT will also be undertaken.

\section{Method}

\subsection{Participants}

Data are collected in the context of the ongoing Brisbane Memory, Attention, and Problem-Solving (MAPS) twin study. Here, we report data from the first 390 twin pairs: $97 \mathrm{MZ}$ females, $87 \mathrm{MZ}$ males, $52 \mathrm{DZ}$ females, $48 \mathrm{DZ}$ males, $106 \mathrm{DZ}$ opposite sex pairs. Most twins had participated in a melanocytic naevi study 2 years earlier (Zhu et al., 1999) and others were ascertained through mail-outs to secondary schools in the Brisbane region. Zygosity was determined by $\mathrm{ABO}, \mathrm{MN}$, and $\mathrm{Rh}$ blood groups and by nine independent polymorphic DNA markers. Twin pairs were excluded if either one had a history of significant head injury, neurological or psychiatric illness, substance dependence, or if they were currently taking long-term medications with central nervous system effects. Participants had normal or corrected-to-normal vision (better than 6/12 Snellen equivalent). The twins were mostly in their penultimate year of secondary school and aged between 15 and 18 years (16.17 years; S.D. $=0.34)$. Written informed consent was obtained from the participant, as well as their parent/guardian, prior to testing.

\subsection{Experimental protocol}

The IT task and IQ test were part of a psychometric battery, which also included a choice RT task and two reading tests. The session approximated $1.5 \mathrm{~h}$ in length, and was either preceded or followed by a testing session of similar duration that involved the measurement of event-related potentials during a delayed response task. Tests were computer administered in the presence of one of three experimenters. One twin completed the psychometric session 
while the other completed the alternate session. The order of session testing was counterbalanced between twin pairs based on the birth order of the twins. A full description of the protocol is given in Wright, Boomsma, et al. (2001).

A subsample of twins (49 pairs) returned for retesting approximately 3 months (1-5 months) after their initial test session. All participants approached for retesting agreed. This sample comprised $23 \mathrm{MZ}$ and $26 \mathrm{DZ}$ pairs (57 females, 43 males). An identical battery of tests was administered on both occasions. To minimise confound effects, the participants performed the sessions in the same order on retest.

\subsection{IT task}

IT was tested by a line discrimination task, which was presented as a pseudocomputer game of choosing the longer of two worms to go fishing. The two lines of comparison were described as worms that would quickly burrow into the ground. The participant's task was to identify the longer worm in an effort to catch the most fish by pressing the corresponding left or right arrow key on the keyboard. Feedback in the form of a fish appeared at the lower lefthand side of the screen following every five correct judgements. Participants were seated approximately $50 \mathrm{~cm}$ from the computer screen. Task instructions were presented on the screen along with several demonstration trials. The importance of accuracy and not reaction time was stressed verbally by the experimenter prior to initialising the task.

The vertical lines measured 22 and $27 \mathrm{~mm}$ in length, were $9 \mathrm{~mm}$ apart, and joined at the top to a horizontal line $12 \mathrm{~mm}$ long (see Fig. 1a). The probability of the longer line appearing on the left or right was equal. The stimulus duration was variable, ranging between 14.2 and $2000 \mathrm{~ms}$. A dynamic mask, consisting of two vertical lines $(37 \mathrm{~mm})$ shaped as lightning bolts (see Fig. 1b), immediately followed the stimulus and was presented for a period of $300 \mathrm{~ms}$ to limit further stimulus processing (Evans \& Nettelbeck, 1993). On each trial, a fixation dot appeared in the centre of the screen for $1 \mathrm{~s}$ (an alerting beep was sounded for $100 \mathrm{~ms}$ at the onset of the dot), followed by a blank screen for $100 \mathrm{~ms}$. The pi figure was then presented, and the participant's first response (left or right) was noted. The screen was blanked for $750 \mathrm{~ms}$ before the next trial was presented. This produced an effect response-stimulus interval of approximately $2 \mathrm{~s}$.

We expected a wide range of IT in our unselected participants and so implemented a Parameter Estimation by Sequential Testing (PEST) procedure (Findlay, 1978; Pentland, 1980)

a)

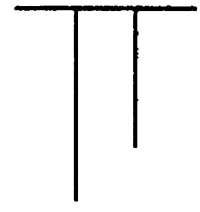

b)

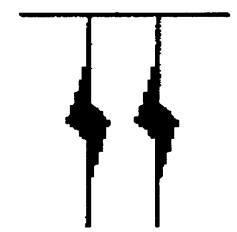

Fig. 1. The stimulus (a) is presented briefly, it is then hidden by a mask (b). 
to allow an efficient estimation from short to long ITs. The PEST estimated IT using a staircase method in which the stimulus duration was altered on the basis of the participant's previous responses. Stimulus durations (stimulus onset asynchronies, SOAs) were defined by the screen refresh rate of $14.2 \mathrm{~ms}$. The PEST procedure began on the fourth trial, as the first three trials were given with long but decreasing durations (113.6 to $56.8 \mathrm{~ms}$ ) to familiarise the participants with the procedure. These three trials were disregarded. For every four correct, consecutive responses the stimulus duration was decreased by the step size, and for every incorrect response the stimulus duration was increased. The step size was dependent on the number of reversals, that is, change in direction from increase to decrease and vice versa of the stimulus duration. After two reversals, the increment reduced to two screen refreshes, $28.4 \mathrm{~ms}$, and following four reversals the step size decreased to one screen refresh. The task would terminate if either one of two conditions was satisfied: the maximum number of trials was presented (110) or the PEST estimates became consistent.

\subsection{IT estimation procedure}

IT was estimated by fitting post hoc a cumulative normal curve (mean $=0)$ to accuracy as a function of SOA. Fig. 2 depicts an individual's data fitted by an ogive where $100 \%$ asymptotic accuracy is obtained. Setting the mean of the cumulative normal to zero sets the accuracy to $50 \%$ at $\mathrm{SOA}=0$ indicating chance accuracy in the absence of a stimulus, and an asymptote of $100 \%$ reflects maximal achievable accuracy at very long SOAs.

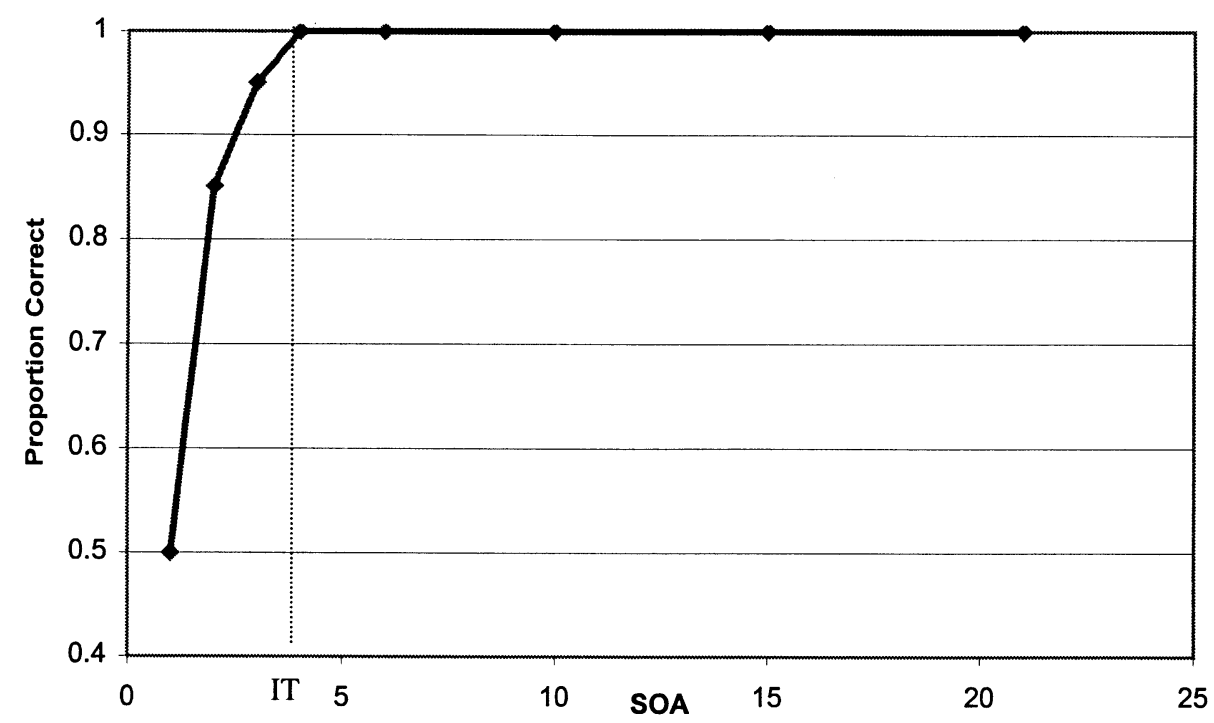

Fig. 2. An individual's data to a cumulative normal curve. At an SOA of zero, the probability of a correct response is .5 and asymptotes to 1 at long SOAs. In this example, chance responding is evident at an SOA of 1 (14.2 ms) and asymptotes at an SOA of $4(56.8 \mathrm{~ms})$; at $95 \%$ accuracy, the SOA is 3 (42.6 ms). 
The statistic of interest is the standard deviation of the curve, which is the SOA at which $84 \%$ accuracy is achieved assuming an asymptote in accuracy of $100 \%$ for each individual. A steeply rising psychometric function will have a lower standard deviation than a more gradually rising function. Multiplying the standard deviation by the appropriate $z$ score will give the SOA at which any arbitrary accuracy is reached (e.g., 1.64 for 95\%). Participants whose data provided a poor fit to the cumulative normal curve $\left(\mathrm{R}^{2}<0.95\right)$ were excluded (i.e., twenty-one participants, or $2.7 \%$ of the sample).

\subsection{Multidimensional Aptitude Battery (MAB)}

A shortened version of the MAB was used, which included three verbal subtests (Information, Arithmetic, Vocabulary) and two Performance subtests (Spatial and Object Assembly). All subtests had a multiple-choice format and were timed at $7 \mathrm{~min}$ each. Participants were not penalised for guessing and were encouraged to answer every item within the time period. Administration and scoring were computerised. Three composite IQ scores were calculated (verbal, performance, full-scale). The MAB was patterned after the WAIS-R and, as a result, possesses good psychometric properties (Jackson, 1984, 1998).

\subsection{Statistical analyses}

Simultaneous equations, established by the known relationship among MZ and DZ co-twins, were applied to the raw data. The equations represented in a classical twin design are $r_{\mathrm{MZ}}=A+C$ and $r_{\mathrm{DZ}}=1 / 2 A+C$, where $A$ represents additive genetic effects and $C$ represents common environment effects. Nonshared environmental effects that include measurement error $(E)$ are not shared by co-twins and are hence absent from the equations. Such a model assumes that genetic, common environment, and unique environment components combine in an additive fashion and that there is random mating in the population (the presence of assortative mating will overestimate $C$ ). Common environment is distributed independently of zygosity and of both genetic and unique environment factors (Hopper, 1993). The total phenotypic variance, in the absence of gene-environment interactions and correlations, is equal to 1 and is expressed as $h^{2}+c^{2}+e^{2}$. These parameters are proportions of genetic, shared environment, and unique environment variance to total phenotypic variance.

Initially, means and variances were tested across birth order and zygosity to assess sampling error. This was done by a maximum likelihood estimation (MLE) procedure using the statistical program Mx 1.50 (Neale, 1997). In this procedure, each of the twin groups (MZ and DZ) has two means (one each for Twins 1 and 2), two variances, and one covariance (a total of 10 parameters). Sex differences were also tested by specifying a female mean and male deviation from that mean but otherwise made no restrictions on the size of variances and covariances of twins (i.e., one additional parameter). Firstly, a saturated model is fitted estimating all 11 parameters, then progressively simplified models are compared to the full model. The fit of each submodel is compared to the one within which it is nested, by a likelihood ratio chi-square test (Neale \& Cardon, 1992). The difference in minus two log likelihood $(-2 \mathrm{LL})$ between the models is compared to the critical value (alpha $=.05)$ of the 
chi-square distribution for the degrees of freedom difference. A nonsignificant chi-square indicates that there is no difference between the full model and the reduced model.

MLEs of the correlations for each zygosity group were computed with means and variances constrained to be equal but including a regression coefficient in the means model for any sex effects (sex differences in the means can inflate twin resemblance for same sex twins). MZ and DZ co-twin correlations were inspected for signs of additive genetic effects (i.e., DZ co-twin correlation is roughly half of the $\mathrm{MZ}$ co-twin correlation).

Before fitting variance components models, we fitted empirical models to obtain MLEs of the phenotypic correlations between the standard deviation of the IT curve (SDIT) and the IQ variables by constraining intraindividual estimates to be the same for Twins 1 and 2 across zygosity groups. In this model we also obtained a single estimate of the total variance for each variable. Subsequently, we fitted models in which we parameterised the total variances and covariances between variables in terms of $A, C$, and $E$. We specified the structure of covariation between IT and IQ variables as a Cholesky decomposition (bivariate: IT and IQ; trivariate: IT, PIQ \& VIQ). This model assumed as many factors for each source of variance $(A, C$, and $E)$ as there were variables. The first factor contained paths leading to all variables in the model, while the next factor contained paths directed to all variables except the first, and, in the trivariate case, the third factor had a single pathway leading to the third variable. Nested $A E$ and $C E$ models were compared to the saturated $A C E$ model using the chi-square difference test. Paths with low factor loadings were also tested for significance in this manner.

\section{Results}

\subsection{Preliminary analyses}

SDIT was normalised by a logarithmic transformation (e.g., Alexander \& Mackenzie, 1992; Crawford, Deary, Allan, \& Gustafsson, 1998; Deary, McCrimmon, \& Bradshaw, 1997). Any score falling outside \pm 3 standard deviations from the mean was considered an outlier and treated as missing: $1.4 \%$ of cases were outlying on first test and two cases on retest. Computer or experimenter error resulted in the loss of IQ data from six participants $(0.76 \%)$ and one person's IT data. Family-wise outliers were further identified in the bivariate and trivariate analyses using the $\mathrm{Mx} \% \mathrm{P}$ function, which calculates likelihood statistics for each family conditional on the model. The output variable of interest was the $z$ score, based on the Mahalanobis distance of the data vectors (each vector represented a twin pair with scores on IQ and SDIT). The $z$ score was assessed retaining the same criterion of \pm 3 standard deviations as a basis for exclusion. Two pairs were outlying in the initial bivariate and trivariate analyses and their scores excluded before the data were reanalysed.

After exclusion of outliers, results from the testing of means and variances across birth order and zygosity were nonsignificant in all models. There were significant sex differences in SDIT and all IQ composite scores. Females were able to discriminate between the lines at a shorter SOA, while males performed better on both scales of the IQ test. Although analyses were conducted on log-transformed SDIT, IT is reported at the 95\% accuracy level for ease of 
interpretation ranging from 42.6 to $712.6 \mathrm{~ms}(157.9 \pm 92.5)$ for females and between 39.4 and $774.1 \mathrm{~ms}(160.2 \pm 126.4)$ for males. The FIQ range for females was 79-141 $(109 \pm 13)$ and for males was 81-145 (113 \pm 13$)$. PIQ and VIQ means for females were $109( \pm 11)$ and 108 $( \pm 10)$, respectively, and for males were $111( \pm 12)$ and $114( \pm 17)$. In all subsequent analyses, sex effects were adjusted for with a multiple regression procedure. The MLE of the test-retest correlation for SDIT was .69 and .90, .87, and .89 for FIQ, PIQ, and VIQ, respectively. Practice effects were demonstrated in SDIT and IQ, with IT (95\% accuracy level) decreasing by $26.9 \mathrm{~ms}$ and FIQ, PIQ, and VIQ increasing by 7, 10, and 4 points, respectively, at the retest.

\subsection{Estimating twin correlations and multivariate model fitting}

MLEs of the phenotypic correlations for MZ and DZ groups for SDIT and FIQ are presented in Table 1 - these are based on data where multivariate outlying twin pairs have been removed. Co-twin correlations for MZs and DZs were, respectively, .34 and .21 for SDIT and .81 and .51 for FIQ, indicating additive genetic effects for both variables, and the possibility of some shared environmental influences (or assortative mating) for FIQ.

The MLE of the phenotypic correlation between SDIT and FIQ was $-.35(N=738)$, indicating that a faster IT was associated with a higher IQ score. The 95\% confidence interval extended from -.28 to -.42 . The correlation between SDIT and PIQ was -.35 , while for VIQ it was -.26 . In accordance with the hypothesis of IT determining IQ and not the reverse, SDIT was entered first into the bivariate and trivariate models. Common environment and additive genetic models were compared by dropping all common environment paths for an $A E$ model and similarly all additive genetic paths for a $C E$ model.

The - 2LL of the fully saturated bivariate model was $5639.358(d f=1500)$. The standardised parameter coefficients from the common factors $(A, C$, and $E$ ) to IT and IQ were, respectively, .57 and $-.48(A), .18$ and $-.33(C), .80$ and $-.02(E)$. Factors influencing only IQ had the following parameter coefficients: .61 $(A), .32(C)$, and $.43(E)$. The change in chi-square was not significant for the $A E \operatorname{model}\left(\Delta \chi_{3}{ }^{2}=3.95, P>.05\right.$, AIC $\left.=-2.05\right)$, but was significant for the $C E$ model $\left(\Delta \chi_{3}{ }^{2}=46.14, P<.05\right.$, AIC $\left.=40.14\right)$, suggesting that additive genes are an important source of variation and covariation between measures and that shared

Table 1

Maximum likelihood estimates of the correlations and 95\% confidence intervals for MZ twins (above diagonal, $n=173-182$ pairs) and DZ twins (below diagonal, $n=196-206$ pairs) for IT and IQ

\begin{tabular}{llllll}
\hline & Twin 1 & & Twin 2 & \\
\cline { 2 - 3 } & IT-1 & IQ-1 & & IT-2 & IQ-2 \\
\hline IT-1 & & $-.32(-.18$ to -.44$)$ & $.34(.21$ to .45$)$ & $-.32(-.18$ to -.45$)$ \\
IQ-1 & $-.36(-.23$ to -.47$)$ & & $-.25(-.12$ to -.37$)$ & $\mathbf{. 8 1}(.76$ to .85) \\
IT-2 & $.21(.06$ to. $\mathbf{3 5})$ & $-.24(-.10$ to -.36$)$ & & $-.27(-.14$ to -.39$)$ \\
IQ-2 & $-.22(-.07$ to -.35$)$ & $\mathbf{. 5 1 ( . 4 1 ~ t o ~ . 6 0 )}$ & $-.46(-.34$ to -.55$)$ & \\
\hline
\end{tabular}

Co-twin correlations for each measure are shown in bold. 
environment is not. Low factor loadings in the full $A E$ model were tested for significance and dropped where appropriate; only the unique environment path between SDIT and FIQ could be dropped. Results of this model are depicted as a path diagram in Fig. 3.

The first additive genetic factor accounted for $36 \%$ of variance in SDIT and $32 \%$ of variance in FIQ, such that genetic influences that decreased SDIT increased FIQ. The genetic correlation between SDIT and FIQ was - .63. FIQ was also influenced by a specific genetic factor that explained a further $49 \%$ of its variance. SDIT was clearly more affected by unique environment (64\% variance) than was FIQ $(19 \%)$. There were no correlated effects of nonshared environment.

The $-2 \mathrm{LL}$ of the fully saturated trivariate model was $11,608.86(d f=2264)$. The change in chi-square was not significant for the $A E$ model $\left(\Delta \chi_{6}{ }^{2}=12.18, P>.05\right.$, AIC $\left.=.18\right)$, but was significant for the $C E$ model $\left(\Delta \chi_{6}{ }^{2}=68.20, P<.05\right.$, AIC $\left.=56.20\right)$. The standardised path coefficients for the base $(A C E)$ model and the reduced $A E$ model (model of best fit) are presented in Table 2.

A common genetic factor mediated the relationship among SDIT (36\%), PIQ (30\%), and VIQ (18\%). The genetic covariance between SDIT and PIQ was larger than that between SDIT and VIQ, as demonstrated by a chi-square difference test, which tested the equality of

Genetic Factors

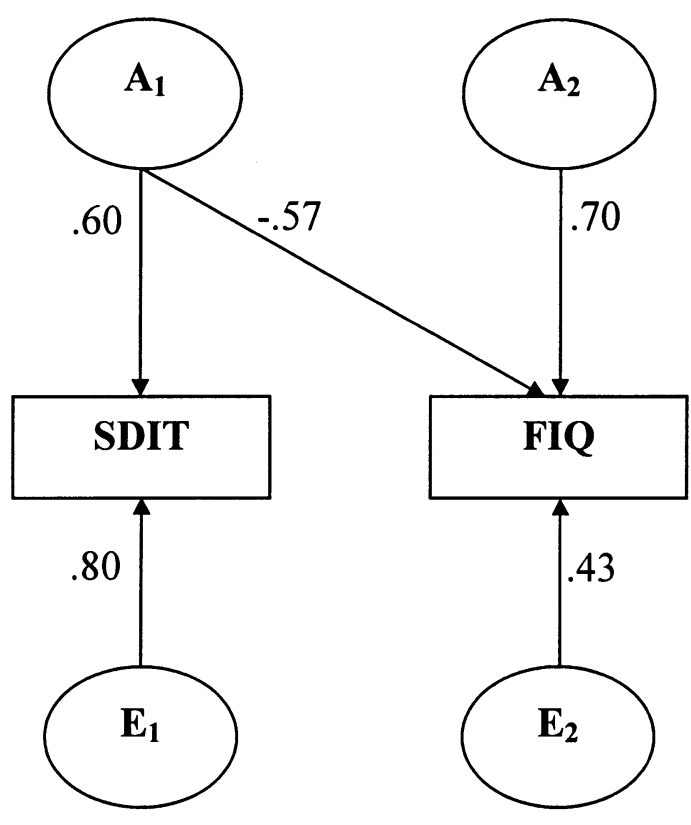

Environmental Factors

Fig. 3. Path diagram showing latent genetic and environmental influences on SDIT and FIQ. 
Table 2

Base model $(A C E)$ and best fitting model (reduced $A E$ ) parameter estimates from latent factors to SDIT, PIQ, and VIQ

\begin{tabular}{rlccccccccc}
\hline & Variable & $A_{1}$ & $A_{2}$ & $A_{3}$ & $C_{1}$ & $C_{2}$ & $C_{3}$ & $E_{1}$ & $E_{2}$ & $E_{3}$ \\
\hline$A C E$ & SDIT & .58 & - & - & -.14 & - & - & .80 & - & - \\
& PIQ & -.47 & .61 & - & .20 & .29 & - & -.06 & .52 & - \\
& VIQ & -.41 & .11 & .53 & .30 & .52 & 0 & .02 & .08 & .42 \\
\multirow{4}{*}{$A E$} & SDIT & .60 & - & - & - & - & - & .80 & - & - \\
& PIQ & -.55 & .65 & - & - & - & - & - & .53 & - \\
& VIQ & -.43 & .35 & .71 & - & - & - & - & .10 & .43 \\
\hline
\end{tabular}

the paths $\left(\Delta \chi_{1}{ }^{2}=4.35, P<.05\right)$. The genetic correlations between SDIT and PIQ was -.65 , while for VIQ it was -.47 . PIQ and VIQ shared a genetic factor that influenced PIQ (42\%) more than VIQ (12\%), and VIQ was influenced by a further genetic factor explaining $50 \%$ of its variance. There were also correlated unique environment effects for PIQ and VIQ.

\section{Discussion}

The significant correlation between IT and psychometric intelligence is well established and replicated in the current study. To date, there has been no attempt to examine whether individual differences in IT display familial aggregation. This study used twins to assess the genetic and environmental variation in IT and its covariance with IQ.

Substantial individual variation in IT and IQ was found in our 16-year-old twin sample. The IT range was wider (higher upper limit) than that reported by previous studies using normal samples (Nettelbeck \& Kirby, 1983; Nettelbeck \& Lally, 1976; Vernon, 1983). Mean IT was in accordance with past estimates, which have ranged from 80 to $228 \mathrm{~ms}$, although the standard deviation was slightly higher than previously reported values, reflecting the wide range of our IT distribution (Mackenzie \& Bingham, 1985; Nettelbeck, 1987). It may surprise some readers that we report IT values as high as $774 \mathrm{~ms}$, within the transformed SDIT distribution such high ITs were not outlying. We tested the fit of the ogive distribution to each individual's data and there were no obvious grounds for excluding participants with these high values. Perhaps these high values reflect the very representative nature of our sample in contrast to the mainly selected samples (of undergraduates) used in much of the IT literature. The IQ range was normally distributed, the mean score was higher than the population mean of 100 .

Variances were equal across birth order and zygosity, with MZ and DZ twin pairs performing similarly for all measures of IT and IQ. Sex effects in IT were minor with the significance of the effect attributed to the exceptional power of the analysis. There was a mean sex difference between males and females in IQ, with males outperforming females. However, because a shortened version of the MAB was used this finding may be artifactual since no sex effects have been reported for MAB IQ. A future analysis of the IQ subtest scores may elucidate the nature of the sex differences found in our sample. 
Practice effects emerged in both measures, this was in the form of a decreased IT and an increased IQ. Findings of improved IT in children and adults on repeated testing have been shown (e.g, Irwin, 1984, exp. 2; Nettelbeck \& Wilson, 1985), although there have also been findings of no practice effects (Irwin, 1984, exp. 3; Vickers, Nettelbeck, \& Willson, 1972); this suggests that particular task parameters may influence this improvement factor. Although there were mean differences on retest, the rank order of individuals was largely maintained, as reflected in the test-retest correlation of .69. This reliability coefficient was within the range of correlations normally reported (Nettelbeck \& Rabbitt, 1992; Sen \& Goswami, 1983; Vernon, 1983) and was most comparable with a study by Larson and Saccuzzo (1989) who used a similar retest interval (1 month), age of participants (19 years), and sample size (220) — retest correlation of .64. Studies of the computerised MAB across time are scarce, but VIQ has been shown to increase on repeated administrations, although PIQ and FIQ were not reported (Harrell, Honaker, Hetu, \& Oberwager, 1987).

Twin correlations for IT demonstrated significant familial aggregation. MZ co-twins showed greater similarity $(r=.34)$ than DZs $(r=.21)$, but this correlation was short of the test-retest correlation indicating that stable features of the unique environment are also important. This is a somewhat unusual finding in that behaviour genetic studies of psychomotor (e.g., motor coordination, pursuit rotor, simple and choice reaction time) and physiological function (e.g., event-related potentials) have shown that MZ co-twin correlations normally approach the test-retest correlation of the measure (e.g., Martin et al., 1985). Unlike psychomotor tasks, IT does not have a motor component (which may account for a substantial proportion of genetic variance in the measure), hence nonshared environment factors must be considered in the individual variation of IT performance. Strategy use in IT has often been an issue of debate (Egan, 1994; Evans \& Nettelbeck, 1993; Knibb, 1992). The finding that the IT-IQ correlation increases when those using strategies are excluded (Mackenzie \& Bingham, 1985; Mackenzie \& Cumming, 1986) may be commensurate with our findings if strategy use is influenced by nonshared environment rather than genes.

Confirming previous studies (Deary \& Stough, 1996; Kranzler \& Jensen, 1989; Nettelbeck, 1987), we found a significant correlation between IT and FIQ $(-.35)$, which was higher for PIQ $(-.33)$ than VIQ $(-.26)$ when these were analysed separately; these were toward the lower range of estimates reported. This study is perhaps the largest study conducted on IT and the unselected nature of our sample suggests that our IT-IQ correlation estimates are unbiased and stable (although this is more apparent in the FIQ correlation, which is a more reliable measure than the subscales).

Bivariate model fitting indicated that the covariance between IT and FIQ was due to a common genetic factor, which explained $36 \%$ of the variance in IT and $32 \%$ of the variance in FIQ. In the trivariate analysis, this factor explained significantly more variance in PIQ (30\%) than in VIQ (18\%). There was no significant common environmental factor. The genetic correlations between IT and each of the IQ measures were higher than the phenotypic correlation (especially for PIQ); indicating that variation in genes that produce faster ITs are strongly related to the variation in genes that promote higher IQs. These findings are in 
agreement with previous genetic studies of information processing variables/factors, which report around half of the variance as genetic (Boomsma \& Somsen, 1991; McGue \& Bouchard, 1989; Rijsdijk et al., 1998; Vernon, 1989; Wright, Smith, Geffen, Geffen, \& Martin, 2000) and with studies that show the processing speed-IQ association is genetically mediated (Baker et al., 1991; Rijsdijk et al., 1998). The results showed that a further genetic factor (explaining $49 \%$ of variance) was needed to explain the genetic effects on FIQ. In the trivariate analysis two genetic factors emerged (one loading on PIQ and VIQ, and the other influencing only VIQ) indicating that different sets of genes (in addition to common genes) affect group factors of intelligence. The remaining variance in IT was composed of nonshared environment, but including a large proportion of measurement error as demonstrated by the imperfect test-retest correlation.

Like others who have investigated elementary cognitive tasks, we have demonstrated that IT shares a substantial genetic relationship with IQ. Furthermore, it was shown that IT shares greater genetic variance with PIQ than VIQ. That different strengths and patterns of genetic and environmental associations have been found across elementary cognitive tasks suggests that there may not be a single processing speed mechanism influencing intelligence but rather different component processes (e.g., speed of perceptual apprehension, speed of short-term memory retrieval), and that the genes influencing these component processes constitute the genes for IQ. Our findings of a stronger PIQ-IT association may be interpreted within this framework such that PIQ involves increased levels of the process tapped by IT than does VIQ (the selection of PIQ and VIQ subtests was based on their low relationships across scales). Crawford et al. (1998) suggested that IT might relate to a spatial orientation and speed process since it is noticed in Performance subtests like Block Design and Object Assembly, which load highly on the perceptual-organisation factor.

While the results appear to favour a component theory of intelligence, where diverse information processes independently contribute to the overall end state of higher cognition (Kranzler \& Jensen, 1991; Sternberg \& Gardner, 1982), the size of the nonshared environmental influences on IT leave open the possibility that genetic overlap between IT and IQ also drives the relationship of other elementary cognitive tasks with IQ. This could be tested by including more tasks in a genetically informed analysis, but rather than using a unitary processing speed factor use individual task measures in the multivariate analysis.

In summary, this study is the first investigation of the possible genetic influences on individual differences in IT and of the extent to which genetic influences mediate its association with psychometric intelligence. The findings provide potentially important information for the biological bases of the information processing-IQ relationship. IT is a basic measure since it assesses speed of perception, which has been linked to rate of sampling information from the environment, without any speed stress on responding. While the genetic influence upon IT is of similar magnitude to that of other elementary cognitive tasks, it is not known whether IT shares the same genetic variance with other information processing measures. This will be a critical issue to broach in future genetic research directed towards lower and high-level information processes. Our larger study that examines several component indices of information processing will address this to some extent using multivariate genetic analysis. 


\section{Acknowledgments}

We greatly appreciate the support from the twins and their parents and for their willingness to participate in this study. We are grateful to Marlene Grace, Ann Eldridge, and Kathleen Moore for the recruitment of twin pairs and data collection. This project is supported by grants from the Australian Research Council (Grant Numbers A79600334 and A79906588) and the Human Frontier Science Program (Grant Number RG0154/1998-B).

\section{References}

Alexander, J. R. M., \& Mackenzie, B. D. (1992). Variations of the 2-line inspection time stimulus. Personality and Individual Differences, 13 (11), 1201-1211.

Baker, L. A., Vernon, P. A., \& Ho, H. (1991). The genetic correlation between intelligence and speed of information processing. Behavior Genetics, 21 (4), 351-367.

Boomsma, D. I., \& Somsen, R. J. M. (1991). Reaction times measured in a choice reaction time and a double task condition: a small twin study. Personality and Individual Differences, 12 (6), 519-522.

Crawford, J. R., Deary, I. J., Allan, K. M., \& Gustafsson, J. (1998). Evaluating competing models of the relationship between inspection time and psychometric intelligence. Intelligence, 26 (1), $27-42$.

Deary, I., McCrimmon, R. J., \& Bradshaw, J. (1997). Visual information processing and intelligence. Intelligence, 24 (3), $461-480$.

Deary, I. J. (1993). Inspection time and WAIS-R IQ subtypes: a confirmatory factor analysis study. Intelligence, $17,223-236$.

Deary, I. J., Caryl, P. G., Egan, V., \& Wight, D. (1989). Visual and auditory inspection time: their interrelationship and correlations with IQ in high ability subjects. Personality and Individual Differences, 10 (5), $525-533$.

Deary, I. J., Head, B., \& Egan, V. (1989). Auditory inspection time, intelligence and pitch discrimination. Intelligence, $13,135-147$.

Deary, I. J., \& Stough, C. (1996). Intelligence and inspection time. American Psychologist, 51 (6), 599-608.

Egan, V. (1994). Intelligence, inspection time and cognitive strategies. British Journal of Psychology, 85, $305-315$.

Evans, G., \& Nettelbeck, T. (1993). Inspection time: a flash mask to reduce apparent movement effects. Personality and Individual Differences, 15 (1), 91-94.

Findlay, J. M. (1978). Estimates on probability functions: a more virulent PEST. Perception and Psychophysics, 23 (2), 181-185.

Harrell, T. H., Honaker, M., Hetu, M., \& Oberwager, J. (1987). Computerized versus traditional administration of the Multidimensional Aptitude Battery - Verbal Scale: an examination of reliability and validity. Computers in Human Behavior, 3, 129-137.

Ho, H., Baker, L. A., \& Decker, S. N. (1988). Covariation between intelligence and speed of cognitive processing: genetic and environmental influences. Behavior Genetics, 18 (2), 247-261.

Hopper, J. L. (1993). Variance components for statistical genetics: applications in medical research to characteristic related to human diseases and health. Statistical Methods in Medical Research, 2, 199-223.

Irwin, R. J. (1984). Inspection time and its relation to intelligence. Intelligence, 8, 47-65.

Jackson, D. N. (1984). Manual for the Multidimensional Aptitude Battery. Port Huron, MI: Research Psychologists Press.

Jackson, D. N. (1998). Multidimensional Aptitude Battery II. Port Huron, MI: Sigma Assessment Systems.

Jensen, A. R. (1998). The $g$ factor. Westport, CT: Praeger.

Kendler, K. S., Neale, M. C., Kessler, R. C., Heath, A. C., \& Eaves, L. J. (1993). A test of the equal-environment assumption in twin studies of psychiatric illness. Behavior Genetics, 23 (1), 21-27. 
Knibb, K. (1992). A dynamic mask for inspection time. Personality and Individual Differences, 13 (2), $237-248$.

Kranzler, J. H., \& Jensen, A. R. (1989). Inspection time and intelligence: a meta-analysis. Intelligence, 13, $329-347$.

Kranzler, J. H., \& Jensen, A. R. (1991). The nature of psychometric g: unitary process or a number of independent processes? Intelligence, 15, 397-422.

Larson, G. E., \& Saccuzzo, D. P. (1989). Cognitive correlates of general intelligence: toward a process theory of $g$. Intelligence, $13,5-31$.

Mackenzie, B., \& Bingham, E. (1985). IQ, inspection time, response strategies in a university population. Australian Journal of Psychology, 37 (3), 257-268.

Mackenzie, B., \& Cumming, S. (1986). How fragile is the relationship between inspection time and intelligence: the effects of apparent-motion cues and previous experience. Personality and Individual Differences, 7 (5), $721-729$.

Martin, N. G., Oakeshott, J. G., Gibson, J. B., Starmer, G. A., Perl, J., \& Wilks, A. V. (1985). A twin study of psychomotor and physiological responses to an acute dose of alcohol. Behavior Genetics, 15 (4), $305-347$.

McGue, M., \& Bouchard, T. J. J. (1989). Genetic and environmental determinants of information processing and special mental abilities: a twin analysis. In: R. J. Sternberg (Ed.), Advances in the psychology of human intelligence 5, (pp. 7-45). Hillsdale, NJ: Lawrence Erlbaum Associates.

Morris, G. L., \& Alcorn, M. B. (1995). Raven's progressive matrices and inspection time: P200 slope correlates. Personality and Individual Differences, 18, 81-87.

Neale, M. (1997). Statistical modeling with Mx. Richmond, VA 23298: Department of Psychiatry, MCV, Box 126.

Neale, M. C., \& Cardon, L. R. (1992). Methodology for genetic studies of twins and families. Dordrecht, Netherlands: Kluwer Academic Publishers.

Nettelbeck, T. (1987). Inspection time and intelligence. In: P. A. Vernon (Ed.), Speed of information-processing and intelligence (pp. 295-346). Norwood, NJ: Ablex Publishing.

Nettelbeck, T., \& Kirby, N. H. (1983). Measures of timed performance and intelligence. Intelligence, 7, 39-52.

Nettelbeck, T., \& Lally, M. (1976). Inspection time and measured intelligence. British Journal of Psychology, 67 (1), 17-22.

Nettelbeck, T., \& Rabbitt, P. M. A. (1992). Aging, cognitive performance, and mental speed. Intelligence, 16, $189-205$.

Nettelbeck, T., \& Wilson, C. (1985). A cross-sequential analysis of developmental differences in speed of visual information processing. Journal of Experimental Child Psychology, 40, 1-22.

Pentland, A. (1980). Maximum likelihood estimation: the best PEST. Perception and Psychophysics, 28 (4), 377-379.

Petrill, S. A., Luo, D., Thompson, L. A., \& Detterman, D. K. (1996). The independent prediction of general intelligence by elementary cognitive tasks: genetic and environmental influences. Behavior Genetics, 26 (2), $135-147$.

Plomin, R. (1986). Behavioral genetic methods. Journal of Personality, 54 (1), 226-261.

Rijsdijk, F. V., Vernon, P. A., \& Boomsma, D. I. (1998). The genetic basis of the relation between speed-ofinformation-processing and IQ. Behavioural Brain Research, 95, 77-84.

Sen, A., \& Goswami, A. (1983). Inspection time across developmental years and its relation to intelligence. Indian Psychologist, 2 (2), 92-99.

Sternberg, R. J., \& Gardner, M. K. (1982). A componential interpretation of the general factor in human intelligence. In: H. J. Eysenck (Ed.), A model for intelligence (pp. 231-254). Berlin: Springer-Verlag.

Vernon, P. A. (1983). Speed of information processing and general intelligence. Intelligence, 7, 53-70.

Vernon, P. A. (1989). The heritability of measures of speed of information-processing. Personality and Individual Differences, 10 (5), 573-576.

Vickers, D., Nettelbeck, T., \& Willson, R. J. (1972). Perceptual indices of performance: the measurement of "inspection time" and "noise" in the visual system. Perception, 1, 263-295. 
Wright, M. J., Boomsma, D., De Geus, E., Posthuma, D., Van Baal, C., Luciano, M., Hansell, N. K., Ando, J., Hasegawa, T., Hiraishi, K., Ono, Y., Miyake, A., Smith, G. A., Geffen, G. A., Geffen, L. B., \& Martin, N. G. (2001). Genetics of cognition: outline of collaborative twin study. Twin Research, 4 (1), 48-56.

Wright, M. J., Smith, G. A., Geffen, G. M., Geffen, L. B., \& Martin, N. G. (2000). Genetic influence on the variance in coincidence timing and its covariance with IQ: a twin study. Intelligence, 28 (4), 239-250.

Zhu, G., Duffy, D. L., Eldridge, A., Grace, M., Mayne, C., O'Gorman, L., Aitken, J. F., Neale, M., Hayward, N. K., Green, A. C., \& Martin, N. G. (1999). A major quantitative trait locus for mole density is linked to the familial melanoma gene CDKN2A: a maximum-likelihood combined linkage and association analysis in twins and their sibs. American Journal of Human Genetics, 65, 483-492 